\title{
Análisis de la demanda de una consulta de Salud Mental coordinada con Atención Primaria
}

\author{
Irene Díaz, Beatriz Mata, Rafael Ángel Baena, Leonor Asensio, Laura Nuevo, \\ y Victoria Muñoz \\ Hospital General Universitario de Ciudad Real (España)
}

\begin{abstract}
Tras la reforma psiquátrica el tratamiento de los pacientes con enfermedad mental se realiza a nivel comunitario, siendo necesaria la coordinación entre Salud Mental y Atención Primaria. Nuestro objetivo es analizar las características de la demanda de Atención Primaria a Salud Mental. Se realiza un estudio observacional, analítico y prospectivo en el que se analizan variables recogidas en una base de datos con formato Access mediante el programa SPSS 19.0. contando con una muestra de 1645 pacientes atendidos en 29 meses, seleccionando aquellos derivados desde Atención Primaria. La distribución fue de un $60 \%$ mujeres y un $40 \%$ hombres, edad media de 48.41 años (desviación típica 18.025). El 40,8\% procedía de Ciudad Real. Derivación más frecuente: Normal (79,8\%). 73\% de hombres y $76 \%$ de mujeres acudieron a cita. El Trastorno Adaptativo fue el más frecuente en ambos sexos, seguido por el Trastorno de ansiedad $(10,2 \%)$ en hombres y Trastorno Depresivo $(17,11 \%)$ en mujeres. Destino al alta más frecuente tras primera consulta: USM $(61,8 \%)$, siendo Trastorno Adaptativo $(24,2 \%)$, Trastorno Depresivo (21,1\%), Trastorno Mixto (12,5\%). Las variables permanecen prácticamente iguales respecto a análisis previos, concluyendo que la coordinación con primaria sigue siendo un objetivo a fomentar para una mejor asistencia a los pacientes.
\end{abstract}

Palabras clave: Salud mental, psiquiatría comunitaria, atención primaria, coordinación.

Analysis of the demand for a mental health consultation coordinated with Primary Care. After the psychiatric reform, the treatment of patients with mental illness is carried out at community level, being necessary the coordination between Mental Health Unit and Primary Care. Our objective is to analyze the characteristics of the demand from Primary Care. An observational, analytical and prospective study is performed in which variables collected in a database with Access format are analyzed through the SPSS 19.0 program counting on a sample of 1645 patients attended in 29 months, selecting those derived from Primary Care. The distribution was $60 \%$ women and $40 \%$ men, mean age of 48.41 years (standard deviation 18,025). $41 \%$ came from Ciudad Real. Most frequent derivation: Normal (79,8\%). $73 \%$ of men and $76 \%$ of women attended an appointment. The Adaptive Disorder was the most frequent in both sexes, being discharged 37,6\%. It was followed by Anxiety Disorder (10,2\%) in men and Depressive Disorder $(17,11 \%)$ in women. More frequent destination after discharge on the first consultation: Mental Health Unit (61,8\%), being Adaptive Disorder (24,2\%), Depressive Disorder (21,1\%), Mixed Disorder $(12,5 \%)$. The variables remain practically the same compared to previous analyzes, concluding that coordination with Primary Care continues to be an objective to be promoted for better patient care.

Keywords: Mental health, community psychiatry, primary care, coordination.

Correspondencia: Irene Díaz Quero. Hospital General Universitario de Ciudad Real. Servicio de Psiquiatría. Calle Obispo Rafael Torija, s/n. C.P.: 13005. Ciudad Real (España). E-mail: idiazq@sescam.jccm.es 
La reforma psiquiátrica realizada en las últimas décadas del siglo pasado supuso una gran revolución a la hora del tratamiento de los pacientes con enfermedad mental, pasando de ser institucionalizados de forma prácticamente indefinida, sin clara mejoría clínica y con el estigma que ello suponía, a ser incluidos dentro de la atención comunitaria y teniendo como vía principal de acceso al sistema sanitario a los especialistas de Atención Primaria (AP en adelante), como el resto de los pacientes de otras especialidades (Moreno-Küstner, 2002).

Este cambio a la hora de tratar a los pacientes con enfermedad mental convertía a los médicos de AP en figuras fundamentales a la hora de la prevención, detección y tratamiento de la patología mental, siendo los que más información pueden aportar del paciente a nivel tanto clínico como psicosocial, contando también para ello con la ayuda de enfermería y trabajadores sociales. Entre un $25 \%$ y un $50 \%$ de la población que es atendida en AP va a presentar un trastorno mental a lo largo de su vida, siendo los más frecuentes los trastornos del estado de ánimo (10\%) y los trastornos de ansiedad (8\%). En cuanto al Trastorno Mental Grave (TMG en adelante), que incluye patologías como la Esquizofrenia, el Trastorno Bipolar o el Trastorno Esquizoafectivo, hasta un 60-65\% de los pacientes que lo presentan no obtienen un tratamiento adecuado, siendo los que más tasa de mortalidad y menor esperanza de vida tienen debido, entre otros, a la comorbilidad con otras patologías (cardiovasculares, oncológicas, respiratorias...), al consumo de tóxicos o por los efectos secundarios de los fármacos (Buitrago et al., 2018).

Por otro lado, en varios estudios realizados se ha visto que una gran parte de los pacientes son derivados por presentar procesos leves o de breve duración acompañados de síntomas depresivos, ansiosos o somatizaciones en relación con situaciones vitales o adaptativas. También se producen derivaciones de pacientes que no presentan síntomas psiquiátricos, perteneciendo a los conocidos como Códigos Z del CIE-10. Estos códigos hacen referencia a los factores que influyen en el estado de salud y contacto con los servicios de salud, y en ellos se incluyen a pacientes que no son diagnosticados de una enfermedad. Estos dos grupos de pacientes pueden ser tratados desde AP mediante la potenciación de los recursos personales, evitando de esta forma el infratratamiento de los TMG, por sobresaturación de los servicios especializados con patología más banal (el denominado "Trastorno Mental Leve"), y la medicalización y psiquiatrización de aspectos de la vida diaria (Buitrago et al., 2018; Díaz, 2011; Lozano, Ortiz, y González, 2014).

Uno de estos estudios fue el llevado a cabo en Asturias, en el cual se analizaban las características de la demanda en SM por parte de diferentes especialidades y la respuesta asistencial dada ante ella. Los resultados obtenidos indicaban que el 77,6\% de la población procedía del medio urbano, el $89,9 \%$ de los pacientes acudieron a la primera consulta y el tipo de consulta solicitada fue ordinaria en un 76,9\% de los casos.

El 95,9\% de los pacientes fueron derivados desde AP y los diagnósticos de derivación más frecuentes fueron Trastorno de Ansiedad (30\%), Trastorno Depresivo 
(23\%) y Trastorno Adaptativo (11,4\%) (Valencia, García, Gimeno, Pérez, y Suárez, 2014).

Para solventar los problemas descritos previamente, se necesitaba una coordinación estrecha entre los servicios de AP y los de Salud Mental (SM en adelante), ya que los médicos de familia son los primeros en entrar en contacto con estos pacientes por su mayor accesibilidad y no suelen contar con una formación específica en este tipo de patologías. Esta coordinación se vio necesaria desde un primer momento y ya quedó establecida en la Declaración Alma-Ata celebrada por la Organización Mundial de la Salud (Organización Mundial de la Salud, 1978). Dado que, como hemos dicho, el enfoque actual de la SM es comunitario, queda sobreentendido que el peso de esta coordinación cae esencialmente sobre los dispositivos por excelencia comunitarios dentro de la red de SM: las Unidades de Salud Mental (o USM) (Fernández, Marín, Leal, y Ballesteros, 2017).

Hay que tener en cuenta que, a la hora de que dos especialistas diferentes traten a un mismo paciente, pueden aparecer algunos problemas: que no coincidan en los objetivos del tratamiento, que la presentación de los síntomas por parte del paciente sea diferente ante cada especialista, o incluso que haya cambios en el tratamiento, falta de adherencia al mismo o factores psicosociales que uno de los dos facultativos desconozca. Para evitar o solucionar estos problemas, es fundamental una buena y frecuente comunicación entre ambos, preferiblemente de forma presencial, aunque cada vez es más frecuente el uso de vías alternativas como la online, respetando siempre la confidencialidad de los datos del paciente. También es importante que ambos médicos se conozcan y sepan cómo trabaja el otro para poder seguir la misma línea terapéutica y complementarse mutuamente (Skodol, 2018).

Dentro de las ventajas que hay al existir esta coordinación podemos encontrar que: se realiza un mejor tratamiento de los pacientes gracias a la mejoría en la prescripción de fármacos, mejora el manejo de los pacientes por parte de AP, se realiza un uso adecuado de los recursos de SM, se reducen los ingresos hospitalarios en unidades de agudos y los pacientes se encuentras más satisfechos (Guilles, Buykx, Parker, y Hetrick, 2015; Skodol, 2018).

Dada la importancia de esta colaboración a la hora del tratamiento de los pacientes, ya se están incluyendo en los diferentes planes de SM de las comunidades autónomas programas de coordinación entre ambos servicios, como es el caso de Andalucía o Castilla-La Mancha. Con estos programas, los médicos de AP son los responsables de atender la mayoría de los trastornos mentales comunes contando con el apoyo de los especialistas de SM (Carmona, 2016; Fernández et al., 2017).

Dentro de este programa de coordinación, contamos en la USM de nuestro hospital con una consulta específica que fue creada en 2015, atendiendo a gran parte de los pacientes derivados desde AP, teniendo como función la coordinación y la recepción 
de la mayor parte de la demanda con esta procedencia. Tras una primera valoración, la psiquiatra encargada de la consulta y coordinación decide el destino del paciente, pudiendo ser éste: alta para seguimiento por AP, continuar con varias consultas previas al alta o pasar al circuito de SM tras la asignación de un psiquiatra o un psicólogo que continuará con el seguimiento. A parte de esta labor, la psiquiatra encargada de la consulta también recibe llamadas telefónicas, correos electrónicos y realiza visitas a los centros de salud del área para conseguir una correcta coordinación con los especialistas de AP, realiza visitas domiciliarias para atender a pacientes que no puedan acudir a la cita (debidamente justificadas) o atiende pacientes que, tras una coordinación con su médico de AP, se considera que han de ser atendidos de manera preferente.

Tras los primeros meses de funcionamiento, se analizaron los datos obtenidos en la consulta, observando que se recibían un alto número de derivaciones erróneas por parte de AP y de otros servicios, lo que hizo preciso el crear unos criterios concretos de derivación y estrechar la coordinación, restringiendo los pacientes recibidos a los derivados por AP y comenzando a concretar aspectos del programa (Mata, López, Rodríguez, y Beato, 2016).

En análisis posteriores se observaron diferentes aspectos, como que un 20,6\% de los pacientes no acudió a la primera consulta (Mata, Nuevo, Asensio, Beato, y Rodríguez, 2017a) o que no se encontraron relaciones significativas entre el lugar de residencia de los pacientes, el centro de salud que realizaba la derivación o las áreas de distribución a las que pertenecían (Mata, Muñoz, Nuevo, López, y Asensio, 2017b).

Respecto a las características de la demanda, el 59,6\% fueron mujeres y el 40,4\% hombres, con una edad meda de 48.66 (desviación típica 18.282) y siendo los diagnósticos más frecuentes: Depresión reactiva (29,4\%), Trastorno Depresivo (18,4\%), Ansiedad (12,2\%), Trastornos mixtos (8,4\%) y Trastornos de Personalidad (7,9\%) (Mata, Nuevo, Asensio, Cabanillas, y Beato, 2017c).

Por último, también se analizaron los factores que se relacionan con la no asistencia a consulta, siendo éstos: sexo, edad, el cese de seguimiento previo por abandono y la existencia de antecedentes psiquiátricos, sin obtenerse una relación estadísticamente significativa en otras variables (Mata et al., 2017d).

Tras estos resultados, quedaba claro que era necesario mejorar la comunicación entre AP y SM para conseguir dar la mejor atención posible a los pacientes en el Centro de Salud, en la USM o en el propio domicilio (Mata et al., 2017a; Mata et al., 2017c; Mata et al., 2017d).

El objetivo de este artículo fue realizar un análisis actualizado de los datos de esta consulta tras un año y medio de funcionamiento de la coordinación presencial, y una vez que se ha dado a conocer el programa a todos los especialistas de AP de la zona. 


\section{MÉTODO}

\section{Participantes}

Se incluyen en el análisis aquellos pacientes que fueron atendidos durante un período de 29 meses, desde Julio de 2015 hasta noviembre de 2017, en nuestra consulta de la USM destinada a la coordinación con AP, contando con una muestra de 1645 pacientes, de los cuales 988 fueron mujeres (60\%) y 657 hombres (40\%), con una edad media de 48.4 años (desviación típica 18). Se excluyeron del análisis aquellos pacientes que eran derivados por error a esta consulta desde otras especialidades.

El tipo de derivación más frecuente fue la normal con un 79,8\%. Del resto, un $18,5 \%$ fue de carácter preferente y un $0,1 \%$ fue urgente, desconociéndose el carácter del resto al no especificarse en la hoja de derivación en soporte digital y el paciente no aportar la hoja en papel. En cuanto a la derivación conjunta a Psiquiatría y Psicología, un 3\% de los pacientes fueron derivados en el mismo acto clínico a ambos profesionales.

Los centros de salud que más derivaciones realizaron fueron los de Ciudad Real capital, sumando un $40 \%$ entre los tres que hay en el municipio, mientras que el resto procedía de las diferentes áreas rurales de la provincia.

El 2016 fue el año con más derivaciones con un 39,5\%, seguido del 2017 con el $31,2 \%$ y del 2015 con el $29,2 \%$.

\section{Instrumentos y variables}

Mediante la realización de una entrevista semiestructurada se recogen las siguientes variables: sexo, edad, antecedentes de seguimiento en Neurología, antecedentes de seguimiento en la Unidad del Dolor y antecedentes de seguimiento previo en Psiquiatría. Respecto a las características de las demandas, se recogen las siguientes variables a través de la hoja de derivación: tipo de derivación (normal, preferente o urgente), si los pacientes son derivados a Psiquiatría y Psicología en el mismo acto clínico (derivación doble), Centro de Salud que deriva, año de la consulta para ver distribución de la demanda en el tiempo $(2015,2016$ o 2017) y el diagnóstico de derivación desde AP. También se recoge si el paciente acude o no a la cita y el destino del paciente una vez se decide el alta. En cuanto a la impresión diagnóstica, se establece tras realizar la valoración psicopatológica de los pacientes.

Se realiza un estudio observacional retrospectivo, descriptivo y analítico.

\section{Análisis de datos}

Las variables son recogidas en una base de datos con formato Access y analizadas mediante el programa Statistical Package for the Social Sciences versión 19.0 (SPSS 19.0). Todos los procedimientos estadísticos se resolverán utilizando un nivel de significación del 5\% (alfa=0,05) e intervalos de confianza del 95\%. En primer lugar, se 
realizó un análisis descriptivo de las variables y de la relación existente entre ellas. En segundo lugar, se utilizaron pruebas para las variables dependientes categóricas (pruebas de independencia de Chi-cuadrado y de Fischer).

\section{Procedimiento}

Todos los pacientes fueron atendidos por la misma profesional que realizó una entrevista clínica semiestructurada y una valoración psicopatológica. A partir de estos instrumentos, se extrajeron las diferentes variables reflejadas anteriormente, complementando algunos datos con la información contenida en la historia digital (volante de derivación desde AP, datos demográficos). Consultado con Comité Ético de Investigación Clínica de nuestro centro, nos informan de que no es precisa autorización expresa dado que se trata de un estudio retrospectivo de tipo epidemiológico.

\section{RESULTADOS}

En cuanto a las variables clínicas analizadas, y respecto a antecedentes de seguimiento en otros servicios, un $11 \%$ de los pacientes de nuestra muestra contaba con antecedentes de seguimiento en Neurología y un $2 \%$ en la Unidad del Dolor.

El diagnóstico principal con el que los pacientes fueron derivados desde AP fue el de Trastorno Depresivo con un 30,1\%, seguido del Trastorno de Ansiedad con un 24,7\% y el Trastorno Adaptativo con un 10,4\%. Otros diagnósticos menos frecuentes son: Trastorno Psicótico (2,3\%), Trastorno por Uso de Sustancias (1,7\%), Trastorno Obsesivo Compulsivo (1,6\%), Trastorno Mental Orgánico (1,5\%), Trastorno Afectivo Bipolar (1\%) y Trastorno de Personalidad (1\%). El 19\% de los pacientes fueron derivados con la categoría "Otros" como diagnóstico.

De todos los pacientes de nuestra muestra, el $75 \%$ acudieron a la cita. De ellos, el $28 \%$ fueron dados de alta tras la primera valoración en la consulta de coordinación, y el 22\% fueron derivados al circuito de SM. En el $9 \%$ fueron precisas varias entrevistas y en el $4 \%$ un breve seguimiento con la psiquiatra coordinadora antes de decidir el destino de los pacientes. El 4\% de los pacientes abandonaron el seguimiento con la psiquiatra coordinadora antes de poder decidir el destino tras la valoración. Al analizar el destino tras la primera consulta según el sexo, observamos que no hay diferencias significativas en los porcentajes entre ambos.

Del 28\% que fueron dados de alta, el $33 \%$ no precisaban de seguimiento en $\mathrm{SM}$ y fueron derivados a AP para continuar con el tratamiento, mientras que el resto fueron dados de alta definitivamente sin necesitar seguimiento por parte de ninguna de las dos especialidades. 
Del $22 \%$ de los pacientes que fueron derivados al circuito de SM, las unidades más frecuentes fueron la USM con un $62 \%$, la Unidad de Conductas Adictivas (UCA en adelante) con el $2 \%$ y la Unidad de Trastornos de la Conducta Alimentaria (UTCA en adelante) con el 1\%. El $2 \%$ de los pacientes fueron derivados a otras especialidades médicas.

Si analizamos esta variable según el sexo, vemos que en ambos el destino más frecuente es la USM con el $62 \%$ en ambos sexos, mientras que en el segundo puesto hay diferencias, siendo lo más frecuente en los hombres la derivación a la UCA con el $4 \%$ y en las mujeres la derivación a otras especialidades con el $2 \%$. Dentro de la red de SM, la UTCA sería el segundo destino más frecuente en las mujeres con un $2 \%$.

En cuanto a la impresión diagnóstica tras la primera valoración, y teniendo en cuenta a toda la muestra, los diagnósticos más frecuentes fueron el Trastorno Adaptativo con el 29,3\%, el Trastorno Depresivo con el 18,5\% y el Trastorno de Ansiedad con el 12,1\%. Otros diagnósticos son: Trastorno Mixto (10,5\%), Trastorno de Personalidad $(8,5 \%)$ y Trastorno Mental Orgánico (4\%).

Gráfico 1. Distribución de la impresión diagnóstica según alta/no alta

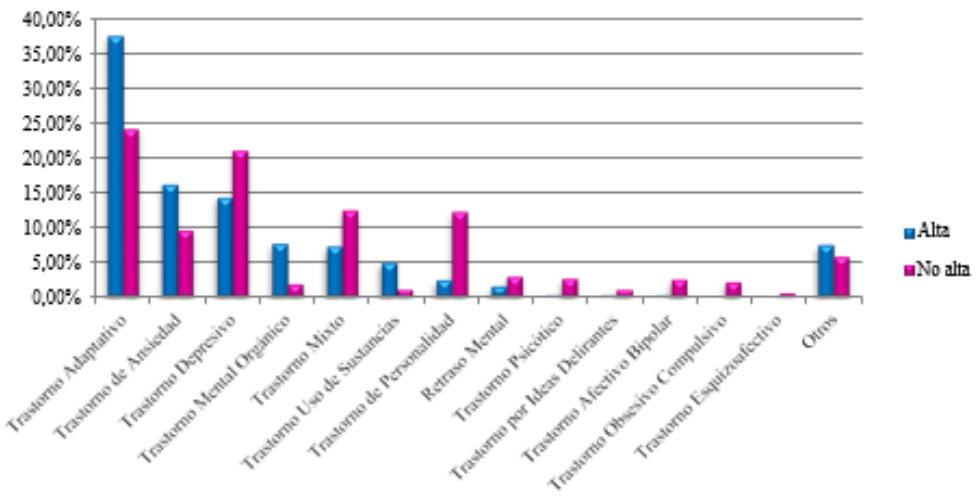

Analizando esta variable según el sexo, el Trastorno Adaptativo se mantiene como el más frecuente en ambos sexos con un $30 \%$ en las mujeres y un $28 \%$ en los hombres. El Trastorno Depresivo es el segundo más frecuente en mujeres con un 17,1\%, seguido del Trastorno Mixto con un 9,7\%, mientras que en los hombres el Trastorno Depresivo pasa a un tercer lugar con un 9\%, estando en segundo lugar el Trastorno de Ansiedad con el 10,2\%.

$\mathrm{Si}$ analizamos la misma variable separando la muestra en los pacientes que son dados de alta y en los que no, observamos que el Trastorno Adaptativo se mantiene como el más frecuente en ambos grupos (el 37,6\% son dados de alta y el 24,2\% permanecen en SM). El Trastorno de Ansiedad es el segundo más frecuente en los 
pacientes dados de alta con un 16,2\% seguido del Trastorno Depresivo con un 14,3\%. En cuanto a los que continúan en SM, el Trastorno Depresivo es el segundo más frecuente con el 21,1\% seguido del Trastorno Mixto (12,5\%) y el Trastorno de Personalidad (12,3\%) (Gráfico 1).

$\mathrm{Al}$ analizar la relación entre acudir a la cita o no y el tipo de derivación, ésta fue estadísticamente significativa $(p<0,001)$, siendo el $74 \%$ de los pacientes derivados como normales los que acuden a la cita frente al $87 \%$ de los preferentes y el $100 \%$ de los urgentes. De la misma forma, la relación entre acudir a cita o no y la existencia de antecedentes de seguimiento previo en psiquiatría también fue estadísticamente significativa $(p<0,001)$, teniendo antecedentes el $82 \%$ de los pacientes que acuden a la cita frente al $69 \%$ que no los tiene. El análisis de la relación entre acudir a cita o no y el sexo, población de origen y el Centro de Salud que realiza la derivación no mostró relaciones estadísticamente significativas.

A continuación, se muestra una tabla resumen donde se recogen los resultados del análisis de las principales variables (Tabla 1) y una segunda tabla en la que se muestran los resultados del análisis según el sexo de las variables más importantes (Tabla 2).

Tabla 1. Resumen de los resultados del análisis de las principales variables

\begin{tabular}{lc}
\hline Diagnóstico de derivación desde AP & Trastorno Depresivo 30\% \\
Trastorno de Ansiedad 24,7\%
\end{tabular}

Tabla 2. Resumen de los resultados del análisis según sexo de las variables recogidas

\begin{tabular}{lcc}
\hline & Hombres & Mujeres \\
\hline \multirow{2}{*}{ Edad } & 44,71 & 50,87 \\
& DS: 17,07 & DS: 18,23 \\
\hline \multirow{2}{*}{ Destino más frecuente al alta } & USM 62\% & USM 62\% \\
& UCA 4\% & Otras especialidades 2\% \\
\hline $\begin{array}{l}\text { Impresión diagnóstica más } \\
\text { frecuente tras primera } \\
\text { valoración }\end{array}$ & Trastorno Adaptativo 28\% & Trastorno Adaptativo 30\% \\
\hline No acuden a cita & $27,2 \%$ & $23,7 \%$ \\
\hline
\end{tabular}

\section{CONCLUSIONES/DISCUSIÓN}

Tras este nuevo análisis observamos escasos cambios en cuanto a las variables demográficas respecto a estudios previos en nuestra área: los porcentajes se mantiene en el $60 \%$ mujeres y $40 \%$ hombres, disminuyendo la edad media a 48,41 , siendo en análisis previos 48.66 (Mata et al., 2017c). 
Respecto a las variables clínicas, se observa un incremento en los pacientes que no acuden a la primera cita, siendo en el nuevo análisis un $25 \%$ cuando en análisis previos eran el 21\% (Mata et al., 2017a). Con los datos obtenidos en cuanto a los destinos tras la primera valoración en consulta en este nuevo análisis, podemos observar que prácticamente la mitad de las derivaciones se resuelven en la primera visita, siendo el $28 \%$ dado de alta y el $22 \%$ entrando directamente en el circuito de SM. De los que son dados de alta en SM, el $67 \%$ no precisa de seguimiento en AP, siendo dados de alta en ambos servicios. Del $22 \%$ que son derivados al circuito de SM, la mayoría pasan a realizar seguimiento en la USM sin ser necesaria la participación de otros dispositivos psiquiátricos, datos que se mantienen en el análisis por sexos, si bien se observa que en caso de ser necesaria la derivación a otros dispositivos, en los hombres es más frecuente que se deriven a la UCA y en las mujeres a la UTCA. Esto puede explicarse por la mayor incidencia de trastornos por uso de sustancias en hombres y de trastornos de la conducta alimentaria en mujeres.

Respecto a los resultados obtenidos en otros análisis como el de Asturias, podemos observar diferencias en el lugar de procedencia de los pacientes, ya que mientras que en nuestra área prevalece la zona rural, en Asturias la mayoría de los pacientes proceden del medio urbano. También se observa una mayor asistencia a la primera consulta que en nuestra área (89,9\% frente al $75 \%)$. En cuanto a los diagnósticos de derivación, observamos que los más frecuentes son Trastorno Depresivo $(30,1 \%)$, Trastorno de Ansiedad (24,7\%) y Trastorno Adaptativo (10,4\%). Comparando estos resultados con los de Asturias, podemos ver que tanto los diagnósticos como los porcentajes son similares, si bien en el análisis de Asturias prevalece el Trastorno de Ansiedad por encima del Depresivo (Valencia et al., 2014).

$\mathrm{Al}$ analizar la impresión diagnóstica observamos un aumento en los casos de Trastorno Adaptativo con un 29,3\% cuando previamente eran el $24,4 \%$ de las derivaciones (Mata et al., 2017c), siendo este aumento mínimo en el resto de los porcentajes: 18,5\% Trastorno Depresivo (antes 18,4\%), 12,1\% Trastorno de Ansiedad (antes 12,2\%), 10,5\% Trastornos mixtos (antes 8,4\%) y 8,5\% Trastornos de Personalidad (antes 7,9\%) (Mata et al., 2017c). En el análisis por sexos se observa que el Trastorno Adaptativo es el más frecuente en ambos. Cabe destacar que la impresión diagnóstica tras la valoración psiquiátrica no coincide con los diagnósticos de derivación emitidos por los médicos de AP, siendo los de éstos: Trastorno Adaptativo 10,4\%, Trastorno Depresivo 30,1\%, Trastorno de Ansiedad 24,7\%, Trastornos mixtos 6\% y Trastornos de Personalidad 1\%.

Como se ha indicado previamente, las discrepancias entre los diagnósticos emitidos por ambos especialistas favorecen tanto la medicalización y psiquiatrización de cuadros reactivos, como el infratratamiento de cuadros graves, apoyando la necesidad de la coordinación entre ambos servicios y la formación de los profesionales de AP con 
el objetivo de mejorar el abordaje de estos pacientes (Díaz, 2011; Mata et al., 2017a; Mata et al., 2017c; Mata et al., 2017d).

Analizando la impresión diagnóstica según el sexo vemos que, salvo el Trastorno Adaptativo, que se mantiene como el primero, en las mujeres el segundo más frecuente es el Trastorno Depresivo mientras que en los hombres es el Trastorno de Ansiedad. Si analizamos los diagnósticos según los que son dados de alta o no, el Trastorno Adaptativo sigue siendo el principal en ambos grupos, seguido del Trastorno de Ansiedad en las altas y del Trastorno Depresivo en los que continúan en SM. Observamos que en ambos grupos (dados de alta y no dados de alta) existen los mismos diagnósticos, aunque con diferente porcentaje, marcando la diferencia en cuanto a su necesidad o no de tratamiento especializado la intensidad o gravedad de la clínica que presenten los pacientes y el manejo de la misma a la hora del tratamiento que tengan desde AP.

Por último, observamos que el tipo de derivación o la existencia de antecedentes de seguimiento en Psiquiatría se relacionan con el acudir o no a la cita, sin obtener una relación estadísticamente significativa en variables como el sexo, la población de origen o el Centro de Salud que realiza la derivación. Estos resultados son similares a los ya obtenidos en estudios previos (Mata et al., 2017b; Mata et al., 2017d).

\section{Conclusiones}

Tras el análisis realizado podemos concluir que se mantienen prácticamente estables las variables demográficas, destacando sólo un aumento en el porcentaje de mujeres y una disminución de la edad media. También se ha producido un incremento en el porcentaje de pacientes que no acuden a la primera consulta.

En cuanto a los diagnósticos, se observa una importante diferencia entre los establecidos por AP y los de SM, y la necesidad de dotar de más recursos asistenciales y, probablemente, clínicos a los profesionales de AP para capacitarlos en el tratamiento de los trastornos más comunes. Para ello, es necesaria la formación de los profesionales de AP y la coordinación entre ambas especialidades, con el objetivo de conseguir una mejor atención y tratamiento integral de los pacientes con estas patologías. Además, consideramos que la supervisión y la posibilidad de coterapia, pueden ayudar a que el profesional de AP pueda manejar casos más limítrofes entre los que requieren o no derivación al especialista. 


\section{REFERENCIAS}

Buitrago, F., Ciurana, R., Chocrón, L., Fernández, M.C., García, J., Montón, C., y Tizón, J.L. (2018). Prevención de los trastornos de la salud mental en atención primaria. Actualización PAPPS 2018. Atención Primaria, 50(1), 83-108. doi: 10.1016/S0212-6567(18)30364-0

Carmona, J. (2016). III Plan Integral de Salud Mental de Andalucía 2016-2020. Sevilla: Junta de Andalucía. Consejería de Salud.

Díaz, D. (2011). Ansiedad, depresión, somatizaciones: proceso asistencial integrado. Sevilla: Junta de Andalucía. Consejería de Salud.

Fernández, J., Marín, M.T., Leal, R., y Ballesteros, J.A. (2017). Plan de Salud Mental de CastillaLa Mancha 2017-2025. Toledo: Servicio Regional de Salud. Consejería de Sanidad.

Guilles, D., Buykx, P., Parker, A.G., y Hetrick, S.E. (2015). Consultation liaison in primary care for people with mental disorders. Cochrane Database of Systematic Reviews, 9. doi: 10.1002/14651858.CD007193.pub2.

Lozano, C., Ortiz, A., y González, C. (2014). Tratamiento y uso de recursos en salud mental de pacientes sin patología. Revista de la Asociación Española de Neuropsiquiatría, 34(122), 267-281. doi: 10.4321/S0211-57352014000200003

Mata, B., López, E., Rodríguez, T., y Beato, L. (2016). Analysis of demand in the first visit to the mental health unit. European Psychiatry, 33S, S559. doi: 10.1016/j.eurpsy.2016.01.1641

Mata, B., Muñoz, V., Nuevo, L., Asensio, L., Beato, L., y Cabanillas, L. (2017a). Related factors with patients who didn't come to visits after being referred by primary care. European Neuropsychopharmacology, 27, S746. doi: 10.1016/S0924-977X(17)31368-8

Mata, B., Muñoz, V., Nuevo, L., López, E., y Asensio, L. (2017b). Analysis of geographical distribution of referrals to psychiatry from primary care. European Psychiatry, S41, S576. doi: 10.1016/j.eurpsy.2017.01.858

Mata, B., Nuevo, L., Asensio, L., Beato, L., y Rodríguez, T. (2017c). Comparison between patients who did not show up for their first visit and the ones who did. European Psychiatry, $41 S$, S576. doi: 10.1016/j.eurpsy.2017.01.857

Mata, B., Nuevo, L., Asensio, L., Cabanillas, L., y Beato, L. (2017d). Descriptive analysis of demand in a mental health-primary care liaison department. European Neuropsychopharmacology, S27, S746. doi: 10.1016/S0924-977X(17)31367-6

Moreno-Küstner, B. (2002). Análisis de patrones de utilización de servicios en pacientes esquizofrénicos. Granada: Universidad de Granada.

Organización Mundial de la Salud (1978). Atención Primaria de Salud. Informe de la Conferencia Internacional sobre Atención Primaria de Salud Alma-Ata, URSS, 6-12 de septiembre de 1978. Ginebra: Organización Mundial de la Salud.

Skodol, A. (2017). Collaboration between prescribing physicians and psychotherapists in mental health care. UpToDate. Waltham, MA: UpToDate Inc

Valencia, F., García, J., Gimeno, A., Pérez, A., y Suárez, M.E. (2014). Análisis de la demanda en Salud Mental en el Principado de Asturias: Características de la demanda y respuestaasistencial. Revista de la Asociación Española de Neuropsiquiatría, 34(124), 741-752. doi: 10.4321/S0211-57352014000400007

Recibido: 26 enero de 2020

Recepción Modificaciones: 18 de febrero de 2020 Aceptado: 20 de febrero de 2020 